\title{
REFERENCES
}

1. A. Frölicher and A. Nijenhuis, Theory of vector-valued differential forms. I, Indag. Math. 18 (1956), 338-359.

2. W. F. Newns and A. G. Walker, Tangent planes to a differentiable manifold, J. London Math. Soc. 31 (1956), 400-407.

3. G. Papy, Sur la définition intrinsèque des vecteurs tangents, C. R. Acad. Sci. Paris 241 (1955), 19-20, ibid. 242 (1956), 1573-1575.

4. H. Whitney, Analytic extensions of differentiable functions defined in closed sets, Trans. Amer. Math. Soc. 36 (1934), 63-89.

UNIVERSITY OF ILLINOIS

\section{THE DUAL OF THE FLABBY IS THE BAR}

JAMES A. SCHAFER

Introduction. The object of this paper is to show the connection between the bar resolution for algebras and the canonical flabby resolution for sheaves. We will show that both resolutions are natural constructions arising from an adjoint relation between two functors. The main result will be that there is a natural chain isomorphism between the "categorical" bar resolution and the dual of the "categorical" flabby resolution.

Throughout this paper $\mathfrak{A}, \mathfrak{B}$ will denote additive categories and $F: \mathfrak{B} \rightarrow \mathfrak{A}, G: \mathfrak{A} \rightarrow \mathfrak{B}$ functors between them with $F$ adjoint to $G$. That is, for each $A$ in $\mathfrak{A}, B$ in $\mathfrak{B}$, there exists a natural isomorphism of abelian groups,

$$
\operatorname{Hom}_{\mathfrak{A}}(F B, A) \cong \operatorname{Hom}_{\mathfrak{B}}(B, G A) .
$$

It follows that there exist natural transformations,

$$
e: I_{\mathfrak{B}} \rightarrow G F, \quad \tilde{e}: F G \rightarrow I_{\mathscr{N}},
$$

with certain properties. An immediate consequence of these properties is that, for all $A$ in $\mathfrak{A}$. we have

$$
G\left(\widetilde{e}_{A}\right) e_{G A}=\operatorname{id}_{G A} .
$$

For this and other results concerning adjoint functors, see [3], [4].

Suppose $S$ and $T$ are functors from the category $\mathfrak{A}$ to the category $\mathscr{B}$ and that $r$ is a natural transformation from $S$ to $T$. If $U$ is a functor from a category $\mathbb{S}$ to a category $\mathfrak{A}$ and $V$ is a functor from a cate-

Received by the editors April 17, 1964. 
gory $\mathfrak{B}$ to a category $\mathfrak{D}$, then we denote by $V * r * U$ the natural transformation from the functor $V S U$ to the functor $V T U$, where $V * r * U(C)=V\left(r_{U C}\right)$. For further details, see [2, Appendix].

The categorical bar construction. Suppose now that $\mathfrak{B}$ possesses cokernels, i.e., every map in $\mathfrak{B}$ has a cokernel, then we may define the "normalized" categorical bar construction as follows.

Let $\bar{F}: \mathfrak{B} \rightarrow \mathfrak{B}$ be the covariant functor defined for each $B$ in $\mathfrak{B}$ by the sequence (2), where $\bar{F} B$ is the cokernel of $e_{B}$,

$$
B \stackrel{e_{B}}{\rightarrow} G F B \stackrel{p_{B}}{\rightarrow} \bar{F} B \rightarrow 0 .
$$

Obviously $\bar{F}$ is a functor and $p: G F \rightarrow \bar{F}$ is a natural transformation. Define the functors $B_{n}: \mathfrak{A} \rightarrow \mathfrak{A}, n \geqq 0$, by

$$
B_{n}=F \bar{F}^{n} G \text {, }
$$

and the natural transformations $s_{-1}: G \rightarrow G B_{0}, s_{n}: G B_{n} \rightarrow G B_{n+1}$, by

$$
\begin{aligned}
s_{-1} & =e * G, \\
s_{n} & =\left(e * \bar{F}^{n+1} G\right)\left(p * \bar{F}^{n} G\right), \quad n \geqq 0 .
\end{aligned}
$$

It has been shown $[5$, p. 271] that there exist unique natural transformations $\epsilon: B_{0} \rightarrow I_{\mathscr{N}}, \partial_{n}: B_{n} \rightarrow B_{n-1}, n \geqq 1$, such that, for each $A$ in $\mathfrak{A}$,

(a) $G\left(\epsilon_{A}\right) s_{-1}=\mathrm{id}_{G A}$,

(b) $s_{-1} G\left(\epsilon_{A}\right)+G\left(\partial_{1}\right) s_{0}=\mathrm{id}_{G B_{0} A}$,

(c) $s_{n-1} G\left(\partial_{n}\right)+G\left(\partial_{n+1}\right) s_{n}=\operatorname{id}_{G B_{n} A}, \quad n \geqq 1$.

To show that this is the bar construction in the usual case, all that one needs to do is to let $\mathfrak{B}$ be the category of modules over a commutative ring $K$; let $\mathfrak{A}$ be the category of $U$-modules, where $U$ is an algebra over $K$; let $G$ be the forgetful functor; and let $F$ be $U \otimes_{K}$.

The categorical flabby resolution. Assume that $\mathfrak{A}$ possesses kernels. An easy exercise concerning adjoints shows that $G$ preserves kernels, i.e., if $f$ is a map in $\mathfrak{A}$, then $G(\operatorname{ker} f)$ is naturally equivalent to $\operatorname{ker} G(f)$.

Define a functor $\widetilde{F}: \mathfrak{A} \rightarrow \mathfrak{A}$ by the sequence (5), where $\widetilde{F} A$ is the kernel of $\tilde{e}_{A}$,

$$
0 \rightarrow \widetilde{F} A \stackrel{\tilde{p}_{A}}{\rightarrow} F G A \stackrel{\tilde{e}_{A}}{\rightarrow} A .
$$

Obviously $\tilde{F}$ is a functor and $\tilde{p}: \tilde{F} \rightarrow F G$ is a natural transformation. Define the functors $\widetilde{B}_{n}: \mathfrak{A} \rightarrow \mathfrak{A}, n \geqq 0$, by

$$
\tilde{B}_{n}=F G \tilde{F}^{n} \text {, }
$$

and the natural transformations $\tilde{\epsilon}: \widetilde{B}_{0} \rightarrow I_{\mathscr{Q}}, \tilde{\partial}_{n}: \widetilde{B}_{n} \rightarrow \widetilde{B}_{n-1}$, by 


$$
\begin{aligned}
\tilde{\epsilon} & =\tilde{e} \\
\tilde{\partial}_{n} & =\left(\tilde{p} * \tilde{F}^{n-1}\right)\left(\tilde{e} * \tilde{F}^{n}\right), \quad n \geqq 1 .
\end{aligned}
$$

It is clear that $\tilde{\partial}_{1} \tilde{\epsilon}=0$ and that $\tilde{\partial}_{n-1} \tilde{\partial}_{n}=0$ so that for each $A$ in $\mathfrak{U},(\tilde{B}(A), \tilde{\partial})$ is an augmented complex over $A$.

To show that this agrees with the dual of the canonical flabby resolution for sheaves, we dualize the above construction and let $\mathfrak{B}$ be the sheaves over $X_{d}$, where $X_{d}$ is the set of the topological space $X$ with the discrete topology; let $\mathfrak{A}$ be sheaves over $X$ and $G=i^{*}$, $F=i_{*}$, where $i$ is the inclusion map of $X_{d}$ into $X$. Then since $\operatorname{Hom}_{X_{d}}\left(i^{*} \mathcal{F}, \mathcal{G}\right)=\operatorname{Hom}_{X}\left(\mathcal{F}, i_{*} \mathcal{G}\right)$, where $\mathcal{F}$ is a sheaf on $X, \mathcal{G}$ is a sheaf on $X_{d}$, the construction above yields the usual flabby resolution for sheaves. For details, see [2].

MAIN THEOREM. Suppose that both the above constructions can be defined (for example, if both categories are abelian), then there exists a natural chain isomorphism $f:(B, \partial) \rightarrow(\widetilde{B}, \tilde{\partial})$.

Lemma. The functors $\bar{F} G$ and $G \tilde{F}: \mathfrak{A} \rightarrow \mathfrak{A}$ are naturally equivalent.

Proof. For each $A$ in $\mathfrak{A}$, we will construct an equivalence $w_{A}: \bar{F} G A$ $\rightarrow G \tilde{F} A$. The construction will be manifestly natural.

Consider the sequence (2) applied to $G A$ in $\mathfrak{B}$. By (1) there exists a map $G\left(\widetilde{e}_{A}\right)$ such that $G\left(\tilde{e}_{A}\right) e_{G A}=\mathrm{id}_{G A}$. Since $\bar{F} G A$ is the cokernel of $e_{G A}$, there exists a map $\mu_{A}: \bar{F} G A \rightarrow G F G A$ such that we obtain the direct sum diagram,

$$
G A \underset{G\left(\tilde{e}_{A}\right)}{\stackrel{e_{G A}}{\rightleftarrows}} G F G A \underset{\mu_{A}}{\stackrel{p_{G A}}{\leftrightarrows}} \bar{F} G A
$$

Applying $G$ to the sequence (5) and using remark (1) again shows that there exists a map $\lambda_{A}: G F G A \rightarrow G \widetilde{F} A$ such that we obtain the direct sum diagram,

$$
G \tilde{F} A \underset{\lambda_{A}}{\stackrel{G\left(\tilde{p}_{A}\right)}{\rightleftarrows}} G F G A \underset{e_{G A}}{\stackrel{G\left(\widetilde{e}_{A}\right)}{\rightleftarrows}} G A .
$$

(Recall that $G$ preserves kernels.) Obviously $\mu_{A}, \lambda_{A}$ define natural transformations $\mu: \bar{F} G \rightarrow G F G$ and $\lambda: G F G \rightarrow G \widetilde{F}$. Let $w=\lambda \mu: \bar{F} G \rightarrow G \widetilde{F}$. Then $w$ is a natural equivalence with inverse $(p * G)(G * \tilde{p})$. This last statement follows from the fact that $p \cdot e=\tilde{e} \cdot \tilde{p}=0$ and that (8) and (9) are direct sum diagrams.

Proof of Theorem. The natural transformations $f_{n}: B_{n} \rightarrow \widetilde{B}_{n}$, $n \geqq 0$, are defined by iterated use of the natural transformation $w$ as follows: 


$$
\begin{aligned}
& f_{0}=\mathrm{id} \\
& f_{n}=\left(F * w * \tilde{F}^{n-1}\right)\left(F \bar{F} * w * \widetilde{F}^{n-2}\right) \cdots\left(F \bar{F}^{n-2} * w * \tilde{F}\right)\left(F \bar{F}^{n-1} * w\right) .
\end{aligned}
$$

Since $B_{n}=F \bar{F}^{n} G$ and $\widetilde{B}_{n}=F G \tilde{F}^{n}$, this amounts to replacing the factor $\bar{F} G$ and $G \tilde{F}$ one step at a time by means of the lemma. Clearly $f_{n}$ is a natural isomorphism with inverse $f_{n}^{-1}$ given by replacing $w$ by $w^{-1}$ in (10) and taking the composite in the opposite order. By means of this isomorphism we may define a boundary $\partial_{n}: B_{n} \rightarrow B_{n-1}$ and an augmentation $\epsilon: B_{0} \rightarrow I_{\mathfrak{A}}$. We will show that this boundary satisfies (a), (b) and (c) for the contracting homotopy $s$ and hence is the "correct" boundary for the bar construction. The definition of $\partial_{n}$ will automatically make $f$ a chain map. Let

$$
\begin{aligned}
\boldsymbol{\epsilon} & =\tilde{\boldsymbol{\epsilon}}, \\
\partial_{n} & =\bar{f}_{n-1}^{-1} \cdot \tilde{\partial}_{n} \cdot f_{n}, \quad n \geqq 1 .
\end{aligned}
$$

The verification of (a), (b) and (c) will follow immediately by induction and a few remarks.

(i) For all $A$ in $\mathfrak{A}$,

(a) $f_{n}(A)=f_{n-1}(\tilde{F} A) F \bar{F}^{n-1}\left(w_{A}\right), \quad n \geqq 1$,

(b) $f_{n}^{-1}(A)=F \bar{F}^{n-1}\left(w_{A}^{-1}\right) f_{n-1}^{-1}(\tilde{F} A), \quad n \geqq 1$.

The proof is an immediate consequence of the definition of $f_{n}$ and the fact that $\widetilde{F}^{n}=\widetilde{F}^{n-1} \cdot \tilde{F}$.

(ii) For all $A$ in $\mathfrak{A}$,

(a) $\tilde{\partial}_{1}(A)=\tilde{p}_{A} \tilde{\epsilon}(\widetilde{F} A)$,

(b) $\tilde{\partial}_{n}(A)=\tilde{\partial}_{n-1}(\tilde{F} A), \quad n \geqq 2$.

This follows immediately from the definitions.

(iii) For all $A$ in $\mathfrak{A}$,

(a) $s_{0}(A)=G F\left(w_{A}^{-1}\right) s_{-1}(\tilde{F} A) w_{A} p_{G A}$,

(b) $s_{n}(A)=G F \bar{F}^{n}\left(w_{A}^{-1}\right) s_{n-1}(\tilde{F} A) G F \bar{F}^{n-1}\left(w_{A}\right), \quad n \geqq 1$.

We give here a proof for (b) since a similar, shorter proof will work for (a). Consider the following diagram.

$$
\begin{aligned}
& G F \bar{F}^{n} G A \stackrel{G F \bar{F}^{n-1}\left(w_{A}\right)}{\longrightarrow} G F \bar{F}^{n-1} G \tilde{F} A \\
& \downarrow p \bar{F}^{n} G A \quad \downarrow p \bar{F}^{n-1} G \widetilde{F} A \\
& \bar{F}^{n+1} G A \stackrel{\bar{F}\left(\bar{F}^{n-1}\left(w_{A}\right)\right)}{\longrightarrow} \bar{F}^{n} G \tilde{F} A \\
& \underset{\downarrow}{|l| \bar{F}^{n+1} G A} \underset{G F \bar{F}^{n+1} G A \stackrel{\downarrow F \bar{F}^{n}\left(w_{A}\right)}{\longrightarrow} G F \bar{F} G \tilde{F} A .}{\longrightarrow}
\end{aligned}
$$


The top diagram commutes since $p$ is a natural transformation from $G F$ to $\bar{F}$, and $\bar{F}^{n-1}\left(w_{A}\right)$ is a map from $\bar{F}^{n-1}(\bar{F} G A)$ to $\bar{F}^{n-1}(G \tilde{F} A)$. The lower diagram commutes since $e$ is a natural transformation from $I_{\mathfrak{B}}$ to $G F$, and $\bar{F}^{n}\left(w_{A}\right)$ is a map from $\bar{F}^{n+1} G A$ to $\bar{F}^{n} G \tilde{F} A$. The lefthand side is by definition $s_{n}(A)$ and the right-hand side is $s_{n-1}(\tilde{F} A)$, whence the conclusion since the bottom map is an isomorphism.

We are now in a position to verify (a), (b) and (c). These are straightforward calculations involving no difficulties. We give for clarification a sample, the inductive step of (c). For simplicity of notation, we write $G F \bar{F}^{n}\left(w_{A}\right)$ as ${ }^{n} w_{A}$, and for a natural transformation $r$, let $r(A)=r, r(\widetilde{F} A)=r^{*}$. Then, for example, remark (iii, (b)) takes the form,

$$
s_{n}={ }^{n} w_{A}^{-1} \cdot s_{n-1}^{*} \cdot{ }^{n-1} w_{A} .
$$

Now suppose (c) true for all $j<n$ and all $A$ in $\mathfrak{A}$, then

$$
\begin{aligned}
& s_{n-1} G\left(\partial_{n}\right)+G\left(\partial_{n+1}\right) s_{n}={ }^{n-1} w_{A}^{-1} \cdot s_{n-2}^{*} \cdot{ }^{n-2} w_{A} \cdot G\left(f_{n-1}^{-1}\right) G\left(\tilde{\partial}_{n}\right) G\left(f_{n}\right) \\
& +G\left(f_{n}^{-1}\right) G\left(\tilde{\partial}_{n+1}\right) G\left(f_{n+1}\right) \cdot{ }^{n} w_{A}^{-1} \cdot s_{n-1}^{*} \cdot{ }^{n-1} w_{A}={ }^{n-1} w_{A}^{-1} \cdot s_{n-2}^{*} \cdot{ }^{n-2} w_{A} \\
& \quad{ }^{n-2} w_{A}^{-1} \cdot G\left(f_{n-1}^{-1 *}\right) G\left(\tilde{\partial}_{n-1}^{*}\right) G\left(f_{n-1}^{*}\right) \cdot{ }^{n-1} w_{A}+{ }^{n-1} w_{A} G\left(f_{n-1}^{-1 *}\right) G\left(\tilde{\partial}_{n}^{*}\right) G\left(f_{n}^{*}\right) \\
& \cdot{ }^{n} w_{A} \cdot{ }^{n} w_{A}^{-1} \cdot s_{n-1}^{*} \cdot{ }^{n-1} w_{A}={ }^{n-1} w_{A}^{-1}\left(s_{n-2}^{*} G\left(\partial_{n-1}^{*}\right)+G\left(\partial_{n}^{*}\right) s_{n-1}^{*}\right)^{n-1} w_{A}=\text { id }
\end{aligned}
$$

by induction applied to $\widetilde{F} A$.

\section{REFERENCES}

1. P. Gabriel, Des catégories abeliennes, Bull. Soc. Math. France 90 (1962), 323448.

2. R. Godement, Theorie des faisceaux, Hermann, Paris, 1958.

3. D. M. Kan, Adjoint functors, Trans. Amer. Math. Soc. 87 (1958), 294-329.

4. S. MacLane, Categorical algebra, Colloquium Lectures at Boulder, Colorado, August 27-30, 1963.

5. - Homology, Academic Press, New York, 1963.

University of Chicago 